\title{
Relationship between blood glucose level and preoperative anxiety and postoperative pain in children (5-15 years old) undergoing adenotonsillectomy
}

\section{Mojtaba Meybodian}

shahid sadoughi university of medical sciences

\section{Mohammad Ansari}

shahi sadoughi university of medical sciences

Mohammadhossein Dadgarnia

shahid sadoughi university of medical sciences

Sedighe Vaziribozorg ( $\nabla$ s.vaziribozorg1408@gmail.com )

shahid sadoughi university of medical sciences https://orcid.org/0000-0001-7749-6196

\section{Original Article}

Keywords: Adenotonsillectomy, Blood glucose, Anxiety, Pain, Children

Posted Date: February 1st, 2021

DOI: https://doi.org/10.21203/rs.3.rs-175227/v1

License: (c) (i) This work is licensed under a Creative Commons Attribution 4.0 International License.

Read Full License 


\section{Abstract \\ Introduction:}

The aim of this study was to determine the relationship between blood glucose level and preoperative anxiety and postoperative pain in children undergoing adenotonsillectomy.

\section{Material and methods}

In this cross-sectional study 156 children undergoing adenotonsillectomy involved. Patients' blood glucose levels were measured at different times. In order to assess the anxiety and restlessness of children, the Anxiety Scale of m-YPAS (Modified Yale Preoperative Anxiety Scale) was used. To evaluate the children's postoperative pain, VAS (visual analog scale) was used before feeding after the surgery.

\section{Results}

Mean of patients' blood glucose levels was $(92.63 \pm 9.33) 15$ minutes before the surgery, $(87.26 \pm 8.32)$ 15 minutes after the surgery and $(85.17 \pm 1.3)$ before feeding after the surgery. The results showed no significant correlation between mean anxiety score and mean blood glucose level at different times. There was a significant correlation between mean pain score and blood glucose level measured 15 minutes after the surgery ( $p$-value $=0.004)$ but showed no significant correlation 15 minutes before the surgery $(p$-value $=0.29)$ and also before feeding after the surgery ( $p$-value $=0.240)$.

\section{Conclusion}

According to our results, since there was only a significant correlation between mean pain score and blood glucose level measured 15 minutes after the surgery (not between the other different times) and over time, due to the body's natural analgesic mechanisms this effect is reduced, it can be concluded that it is not rational to advise hyperglycemic solutions and serums containing glucose in children undergoing adenotonsillectomy.

\section{Introduction}

Adenotonsillectomy is a common surgical procedure for treating obstructive sleep apnea and tonsillitis. This surgery is one of the most common surgical procedures performed on children in the United States. Surgeries such as adenotonsillectomy are stressful experiences for children. Hospitalization creates a social crisis for children and requires child adaptation to the new environment; on the other hand, they suffer from a disease and exposed to unknown and hazardous factors $(1,2)$. Anxiety is a natural adaptive response to stress-induced surgery and may occur at any time during the preoperative period. Patients start feeling anxious when being aware of the need for surgical treatment and their anxiety reach 
its maximum during hospitalization; it can even cause symptoms such as distress, restlessness, fear, and palpitations. Therefore, anxiety is a potential risk to the child and can endanger his/her health (3). Preoperative anxiety not only heightens the pain in children with surgical experience but also has a negative effect on postoperative recovery and possibly postoperative period. Anxiety-induced surgery leads to negative behaviors such as restlessness, crying, and urinary incontinence during surgical experience; and it is associated with a number of maladaptive behaviors such as increased distress in the recovery room, eating disorders, sleep disturbances, and nocturnal enuresis after surgery. Reducing anxiety brings about faster and better recovery, less anesthetic use, better pain tolerance, early discharge, and ultimately less postoperative complications (4). More than $65 \%$ of children undergoing anesthesia and tonsillectomy surgery show anxiety and restlessness before and during anesthesia; this induced anxiety and restlessness can interfere with the natural process of anesthesia and can even reduce the oxygen saturation. Over the years, many efforts have been made to reduce pre-operative anxiety and restlessness and post-operative pain especially in children. Because of the necessity of preparations before elective surgeries; that is the necessity of child's NPO (non per oral), the presence of surgical stress, and body responsiveness promoting body defense, there is a probability for hypoglycemia before or after anesthesia, which can heighten the stress and the restlessness of the patient, resulting in postoperative pain and other complications and later recovery of the patient. In addition, the administration of general anesthetic drugs such as opiates and Thiopental can undermine the response to surgical stress and cause hypoglycemia (5). Some studies showed that preoperative glucose administration can decrease postoperative pain and shorten recovery and length of stay in hospital (6). The aim of this study was to determine the relationship between blood glucose level and preoperative anxiety and postoperative pain in children undergoing adenotonsillectomy in order to reduce anxiety and postoperative pain in these children.

\section{Methods}

After approval by the ethics committee and obtaining written informed consent, in this cross-sectional study 156 children aged between 5 to 15 years old undergoing adenotonsillectomy were involved. Children with a history of any diseases including diabetes, kidney diseases and any disorders affecting on detecting glucose level by a glucometer (such as bilirubin $>20$ and $\mathrm{Hct}<20$ or $\mathrm{Hct}>65 \mathrm{mg} / \mathrm{dl}$ ) and patients who did not accept to participate in this study, were excluded. All patients fasted for 8 hours. In order to assess the anxiety and restlessness of children, the Anxiety Scale of m-YPAS was used 15 minutes before the surgery (Fig. 1). This scale consists of 5 items and 22 questions. The scores for each item are added up to obtain child's anxiety score. Fifteen minutes before the surgery, patients' blood glucose level was measured and recorded. Then all patients underwent general anesthesia by the same anesthesiologist. Children underwent adenotonsillectomy by the same surgeon and the same method. Again, blood glucose level was measured 15 minutes after the surgery and also before beginning of the feeding (in the ENT ward), using a glucometer (A quantitative diagnostic kit for glucose determination and an auto-analyzer device that depicts glucose level using glucose kit, was used). To evaluate the 
children's postoperative pain, VAS (Fig .2) was used before feeding after the surgery. All the obtained data was recorded and analyzed by SPSS (version 21). P value $<0.05$ was considered statistically significant.

\section{Results}

In this cross-sectional study, 156 children undergoing adenotonsillectomy were involved. Concerning gender, 83 of the children were boys and 73 were girls. The mean age of the children was $(7.45 \pm 2.16)$ years. Mean of patients' blood glucose levels was $(92.63 \pm 9.33) 15$ minutes before the surgery, $(87.26 \pm$ 8.32) 15 minutes after the surgery and $(85.17 \pm 1.3)$ before feeding after the surgery. The mean anxiety score was $(10.54 \pm 3.76)$ and the mean VAS was 4.17 . The results showed no significant correlation between mean anxiety score and mean blood glucose level 15 minutes before the surgery ( $p$-value = $0.839), 15$ minutes after the surgery ( $p$-value $=0.636)$ and before feeding after the surgery ( $p$-value = 0.757). There was a significant correlation between mean pain score and blood glucose level measured 15 minutes after the surgery ( $p$-value $=0.004)$ but there was no significant correlation between mean pain score and blood glucose level measured 15 minutes before the surgery $(p$-value $=0.29)$ and also before feeding after the surgery ( $p$-value $=0.240$ ).

\section{Discussion}

Adenotonsillectomy is one of the most common surgical procedures performed on children in the United States. Surgeries such as adenotonsillectomy are as stressful experiences for children $(7,8)$. Although the researchers' view on the effects of preoperative anxiety on children and the intra-operative and postoperative outcome, especially on postoperative healing, is different, the majority of them believe that preoperative anxiety at a mild to moderate level can lead to behavioral improvements during the postoperative period, but high anxiety before surgery leads to unpleasant psychological and physiological outcomes. Over the years, many efforts have been made to reduce preoperative anxiety and restlessness, especially in children. Various studies using quantitative and qualitative approaches pointed out the anxiety reduction factors in children $(9,10)$. On the other hand, Pain management after tonsillectomy in children is essential because of the morbidity associated with uncontrolled pain in children and the emotional components of children and their families. Pain treatment after tonsillectomy in children has been regularly discussed for years and different drugs are suggested for this reason(11). However, no studies have yet probed the association between blood glucose level and preoperative anxiety and postoperative pain in children candidates for tonsillectomy. Therefore the aim of this study was to determine the relationship between blood glucose level and anxiety and postoperative pain in children undergoing adenotonsillectomy. We hypothesized that if there is a relationship between blood glucose level and preoperative anxiety and postoperative pain, prescribing of intravenous glucose, can reduce the pain and anxiety in children undergoing adenotonsillectomy. According to our results, there was no significant relationship between preoperative anxiety and blood glucose level. Also in our study, the results of postoperative pain assessment in children using VAS showed no significant relationship between postoperative pain and blood glucose level measured before feeding after the surgery. In a study 
by Samadani et al, the relationship between blood glucose and hemodynamic status of non-diabetic patients undergoing non-emergency surgery was evaluated. They discovered a significant correlation between preoperative blood glucose and diastolic and systolic blood pressure as well as the level of stress suggesting that complications and undesirable hemodynamic changes in patients can be reduced by carefully controlling the blood sugar of the patients before and during the surgery (12). In our study hemodynamic changes were not evaluated. Helminen et al evaluated the effect of preoperative intravenous carbohydrate loading on preoperative discomfort in elective surgery patients. They compared feelings of thirst, hunger, mouth dryness, weakness, tiredness, anxiety, headache and pain of each patient preoperatively using a visual analog scale between three different groups (intravenous $5 \%$ glucose infusion $(1000 \mathrm{ml})$, carbohydrate-rich drink $(400 \mathrm{ml})$ and overnight fasting group). They found that in the fasting group (lowest blood glucose level), thirst, hunger, mouth dryness, weakness, tiredness, and anxiety was significantly increased $(P<0.05)(6)$ but in our study, there was no significant relationship between preoperative anxiety and pain and blood glucose level in children underwent adenotonsillectomy. Louis Hinshaw et al evaluated the efficacy of the intra peritoneal instillation of $5 \%$ dextrose in water (D5W) during radiofrequency ablation of peripheral liver tumors for decreasing post-radiofrequency pain and narcotic use. They found that pretreatment with intra peritoneal D5W before radiofrequency ablation of peripheral liver tumors in adults, decreases pain, narcotic use, and length of hospital stay and they suggested that the effect of pretreatment with D5W is greatest in the first $16 \mathrm{hr}$ after the procedure (13). In our study narcotic use was not evaluated. In a study by Jovanović NC et al, they concluded that $40 \mathrm{ml}$ of $50 \%$ glucose solution given in two identical boluses has good analgesic effects in the treatment of postoperative pain in old oncology patients. The pain was not completely eliminated, but it was significantly decreased and became tolerable (14). Although the administration of glucose has reduced the pain and anxiety of patients undergoing different types of surgeries in previous studies, but as we mentioned before, no study evaluated relation between blood glucose level and preoperative anxiety and postoperative pain in children.

\section{Conclusion}

According to our results, since there was only a significant correlation between mean pain score and blood glucose level measured 15 minutes after the surgery (not between the other different times) and over time, due to the body's natural analgesic mechanisms this effect is reduced, it can be concluded that it is not rational to advise hyperglycemic solutions and serums containing glucose in children undergoing adenotonsillectomy.

\section{Recommendation}

To obtain more reliable and detailed findings on the effect of blood glucose level on postoperative pain and anxiety, future studies are recommended to recruit a larger number of children undergoing different types of surgeries. Moreover, further studies are recommended to evaluate the effects of pretreatment with intravenous glucose on postoperative pain in children. 


\section{Declarations}

* Authors have no conflicts of interest.

* Study protocol was in accordance with the latest Declaration of Helsinki for medical research involving human subjects and was approved by local ethics committee.

This article does not contain any studies with animals performed by any of the authors. $\mathrm{c}$

* Informed consent was obtained from all participants of the study.

\section{References}

1. Özköse Z, Akçabay M, Kemaloğlu YK, Sezenler S (2000) Relief of posttonsillectomy pain with lowdose tramadol given at induction of anesthesia in children. Int J Pediatr Otorhinolaryngol 53(3):207214

2. Vaezzadeh N, Douki ZE, Hadipour A, Osia S, Shahmohammadi S, Sadeghi R (2011) The effect of performing preoperative preparation program on school age children's anxiety. Iranian journal of pediatrics 21(4):461

3. Bahrami N, Soleimani MA, Erjini Z, Shraifnia H, Masoodi R, Shahrokhi A (2012) The effect of nursing process-based care on patients' anxiety of candidates for women's elective surgery. Iran Journal of Nursing 25(77):30-39

4. Reyhani T, Dehghan Z, Shojaeian R, Ashgharinekah S, Behnam Vashani H (2014) The influence of the puppet Kolah Ghermezi on preoperative anxiety among hospitalized children with appendicitis in Dr Shaikh hospital of Mashhad. Evidence Based Care 4(3):77-86

5. Zahedi H, Akhyani V, Khan ZH, Yunesian M (2006) Evaluation of perioperative blood glucose changes during elective eye surgeries under general anesthesia, with two methods: Glucometer and lab assay. Tehran University Medical Journal TUMS Publications 64(3):44-51

6. Helminen H, Viitanen H, Sajanti J (2009) Effect of preoperative intravenous carbohydrate loading on preoperative discomfort in elective surgery patients. Eur J Anaesthesiol 26(2):123-127

7. Ferkol T, Schraufnagel D (2014) The global burden of respiratory disease. Annals of the American Thoracic Society 11(3):404-406

8. Steele MP, Brown KK (2007) Genetic predisposition to respiratory diseases: infiltrative lung diseases. Respiration 74(6):601-608

9. Mandal A, Kabra SK, Lodha R (2015) Upper Airway Obstruction in Children. Indian J Pediatr 82(8):737-744

10. Linscott MS, Horton WC (1979) Management of upper airway obstruction. Otolaryngol Clin North Am 12(2):351-373

11. Alm F, Jaensson M, Lundeberg S, Ericsson E (2017) Adherence to Swedish guidelines for pain treatment in relation to pediatric tonsil surgery: a survey of the multidisciplinary team. Int $\mathrm{J}$ Pediatr 
Otorhinolaryngol 101:123-131

12. Samadanifard A, Arshad M, Rajabtaragi M, Setayesh H (2015) Comparison of hemodynamic state and serum glucose in non-diabetic patients during non-emergency surgery. Razi Journal of Medical Sciences 22(132):87-94

13. Hinshaw JL, Laeseke PF, Winter TC III, Kliewer MA, Fine JP, Lee FT Jr (2006) Radiofrequency ablation of peripheral liver tumors: intraperitoneal $5 \%$ dextrose in water decreases postprocedural pain. American journal of roentgenology 186(5_supplement):S306-S310

14. Jovanovic NC, Dzodic R, Celebic A, Zegarac M, Djurisic I, Stojiljkovic D (2003) [Treatment of postoperative pain in elderly oncology patients with intravenous administration of a $50 \%$ glucose solution]. Srp Arh Celok Lek 131(1-2):52-54

\section{Figures}




\section{A. Activity \\ B. Vocalizations \\ C. Emotional Expressivity \\ D. State of Arousal \\ E. Use of Parent}

\section{A. Activity}

0. Can't code (child not visible)

1. Looking around, curious, playing with toys, reading(or other age appropriate behavior); moves around holding area/ treatment room to get toys or go to parent: may move toward OR equipment

2. Not exploring or playing, may look down, may fidget with hands or suck thumb (blanket); may sit close to parent while waiting, or play has a definite manic quality

3. Moving from toy to parent in unfocused manner, nonactivity derived movements; frenetic/frenzied movement or play; squirming, moving on table, may push mask away or clinging to parent

4. Actively trying to get away, pushes with feet and arms, may move whole body; in waiting room, running around unfocused, not looking at toys or will not separate from parent, desperate clinging

\section{B. Vocalizations}

0. Can't code (child not visible or can't hear audio)

1. Reading (nonvocalizing appropriate to activity), asking questions, making comments, babbling, laughing, readily answers questions but may be generally quiet; child too young to talk in social situations or too engrossed in play to respond

2. Responding to adults but whispers, "baby talk", only head nodding

3. Quiet, no sounds or responses to adults

4. Whimpering, moaning, groaning, silently crying

5. Crying or may be screaming "no"

6. Crying, screaming loudly, sustained (audible through mask)

\section{Emotional Expressivity}

0. Can't code (can't see face or child not visible)

1. Manifestly happy, smiling, or concentrating on play

2. Neutral, no visible expression on face

3. Worried (sad) to frightened, sad, worried, or tearful eyes

4. Distressed, crying, extreme upset, may have wide eyes

\section{State of Apparent Arousal}

0. Can't Code (child not visible)

1. Alert, looks around occasionally, notices watches what anesthesiologist does with him/her (could be relaxed)

2. Withdrawn child sitting still and quiet, may be sucking on thumb or face turned into adult

3. Vigilant looking quickly all around, may startle to sounds, cyes wide, body tense

4. Panicked whimpering, may be crying or pushing others away, turns away

\section{E. Use of Parents}

\section{0 . Can't code (child not visible)}

1. Busy playing, sitting idle, or engaged in age appropriate behavior and doesn't need parent; may interact with parent if parent initiates the interaction

2. Reaches out to parent(approaches parent and speaks to otherwise silent parent), secks and accepts comfort, may lean against parent

3. Looks to parents quietly, apparently watches actions, doesn't seek contact or comfort, accepts it if offered or clings to parent

4. Keeps parent at distance or may actively withdraw from parent, may push parent away or desperately dinging to parent and will not let parent go

Total adjusted score $=(\mathrm{A} / 4+\mathrm{B} / 6+\mathrm{C} / 4+\mathrm{D} / 4+\mathrm{E} / 4) \times 100 / 5$

\section{Figure 1}

\section{Modified Yale Preoperative Anxiety Scale}



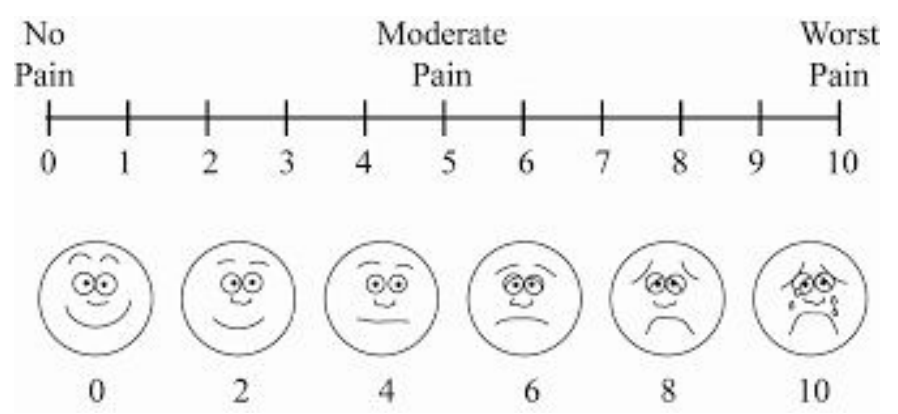

Figure 2

Visual analog scale 